\title{
The Effect of Eccentric Exercise with Variations in Rest Interval on Creatine Kinase and Lactate Dehydrogenase Enzymes in Sedentary Young Men
}

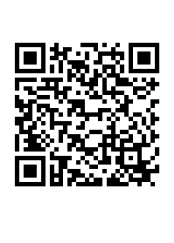

\author{
Hossein Mohammadii* and Alun Williams ${ }^{2}$ \\ ${ }^{1}$ Department of Sport Physiology, University of Birjand, Iran \\ ${ }^{2}$ Department of Sport and Exercise Sciences, Manchester Metropolitan University, UK \\ Submission: November 13, 2019; Published: November 27, 2019 \\ *Corresponding author: Hossein Mohammadi, Department of Sport Physiology, University of Birjand, Iran
}

\begin{abstract}
Various studies have demonstrated that different rest Intervals and set -repetition has a significant effect on hormonal and metabolic responses. This factors can lead to different muscle damage responses. 20 untrained subjects $(25.4 \pm 0.068$ years, height $1.74 \pm 0.97 \mathrm{~cm}$ and weight $30.8 \pm 8.48 \mathrm{~kg}$ ) in three sessions of eccentric resistance exercise with 24 hours rest between each session Participated in this study. Subjects were divided into 2 groups of 10 subjects who performed 50 eccentric contractions with different number of 5 sets, 5 repetitions, and the interset rest interval 1 and 3 minutes with $85 \%$ of one repetition maximum (1RM). Creatine Kinase and Lactate Dehydrogenase were measured immediately before , immediately after each session and 24 hours after the last training session. Variance analysis with repeated measurment and Bonferroni post-hoc test were used for statistical analysis of data. There is no significant difference in Creatine Kinase and Lactate Dehydrogenase between four groups at different time points. $(\mathrm{P}<0.05)$. The repetition of eccentric exercise for three consecutive days causes muscle damage that is independent of manipulating the interset rest intervals.
\end{abstract}

Keywords: Eccentric exercise; Creatine kinase; Lactate dehydrogenase

\section{Introduction}

Participation in unaccustomed, eccentrically biased exercise often results in ultra structural damage to skeletal muscle (Friden \& Lieber 2001; Paul et al. 1989; Friden et al. 1983). Symptoms of this exercise-induced muscle damage (EIMD) include elevated muscle proteins in the blood, delayed-onset muscle soreness, swelling, a decreased range of motion, and impairment of proprioceptive function and neuromuscular control (for a review, see Byrne et al. 2004). One of the consequences of the resistance exercises is the delayed onset muscle soreness. Delayed muscle soreness causes pain, swelling, decreased range of motion and functional strength reduction (Dipasqualeetal. 2011). Eccentric contractions can cause muscle pain. To reduce muscle pain induced by eccentric contractions, add exercise of the lower angular movement can be used in practice, which is not possible for most sports; Also, due to reduced fitness in the off-season, athletes, in order to do pre-season preparation, need to repeat the same exercise intensity and continuously within a few days, and this has caused a lot of athletes and ordinary people to continue to practice while having muscle soreness. It is very important to prevent such damage. Since these injuries are inevitable, providing a treatment to help relieve the discomfort, injury and disability, and fast return of the individual to exercise is very important (Moradi et al., 2002).Warm up before exercise, cool down and stretch afterwards as guidelines for the prevention of delayed onset muscle soreness have been introduced (Kazunori Nosaka et al., 2000).

In addition, other methods of treatment in order to reduce the signs and symptoms of delayed onset muscle soreness have been used. Medications due to their side effects and the possibility of getting their doping agents cannot be an appropriate treatment (J.B. Rodenburg et al., 1994). Non-drug treatments including massage, ice massage, and ultrasound waves, repeated practice cause subcutaneous nerve stimulation (Divakara kedlaya, 2001; G.Howatson,D et al, 2005; Kasonuri Nosaka., 1995). But since the main cause and mechanism of this phenomenon are not known exactly, the results of the application of different methods can be different (Michelle A, et al., 2002).

Repeating this exercise as a method of treatment has been studied on delayed onset muscle soreness. The researchers 
repeated the research on the effects of exercise and have come to the conclusion that the effect of repeated eccentric exercise is similar to the first, markers of muscle damage, primarily due to reduced exercise, Repeating the same exercise the researchers concluded that in the first 3 days, due to a series of adaptations in muscle, injury does not cause deterioration of muscle function (Kasonuri Nosaka et al, 1995; Moradi, 2002). According to studies and eyewitness accounts, team and individual sportsmen in our country, have to practice at least 3 times a week. As the muscle after 48 hours of rest, is not completely returned to the initial state it is not yet clear whether the exercise or performance will decrease in such a situation [1-5].

Rodrigo [6] investigated the changes in cellular injury indices after two eccentric exercises with a 4 week interval with $100 \%$ intensity of one maximum repetition with a difference in contraction. The results of this study showed that the number of contractions versus shift-repetition manipulation is more effective than muscle damage. Maeiyo et al. [3] reported that creatine kinase enzyme activity was more than a three minute interval after a resistance exercise with an intensity of $65 \%$, a maximum repetition of 10 set with 10 repetitions, and a one minute resting distance. On the other hand, Roberio et al. [6] did not show any significant difference in creatine kinase and lactate dehydrogenase enzyme activity after three set and ten repetitions. Given that even if the total number of eccentric contractions in a similar exercise is similar, the magnitude of muscle damage is likely to vary with the simultaneous manipulation of the number of sets, the number of repetitions, and the rest time between the exercise times, and given that most Past research has looked at an outsourcing exercise that is less like an athlete training program; it's a question of which rest time (one minute to three minutes) and how many times and again it leads to less muscle damage?

\section{Methods}

20 subjects were selected randomly and divided into two groups of 10: the first group was rested one minute and five set with 10 repetitions; the second group was resting for three minute and five set with ten replications. In the present study delayed onset muscle pain free weights (dumbbells) were used. Because, eccentric contractions may cause delayed muscle aches, lifting the second phase, which involves reducing weight with the non-dominant hand was used (Kasonuri Nosaka., 1995). In order to become familiar with the test and the $1 \mathrm{RM}$, subjects were recruited through questionnaires 48 hours before the start of the performance were invited to the Physical Education School Hall. During their meeting, they start to warm up for 5 minutes. After warming up, the estimated 1RM, with 3 to 5 repetition, the maximum concentric contraction was in the non-dominant hand (Shahbazpour, N., 2004). 48 hours after the test, measurements were performed in the period between $8 \mathrm{pm}$ to $11 \mathrm{pm}$. Subjects initially completed questionnaire and consent form.

Questions were as follows: Participants should not experience neurological, muscle, heart and brain disease, and the history of the non-dominant upper limb fractures were included. During the six months prior to the study, the nondominant upper extremity weight training Companies had drug injection and during the 10 days prior to study, by the onset of analgesic or pain or discomfort in the non-dominant upper limbs did not. They did not have high- fat meal, especially during the protocol and the night before each workout had to begin. Height, weight, and blood samples for determination of creatine kinase and lactate dehydrogenase levels were measured [6-9] (Sample Table).

\begin{tabular}{|c|c|c|}
\hline Name: & Yes & No \\
\hline the history of the non-dominant upper limb fractures & & \\
\hline experience neurological, muscle, heart and brain disease & & \\
\hline $\begin{array}{c}\text { During the six months prior to the study, the non- } \\
\text { dominant upper extremity weight training }\end{array}$ & & \\
\hline drug injection and during the 10 days prior to study & & \\
\hline
\end{tabular}

For the measurement of Creatine Kinase and lactate dehydrogenase levels, $5 \mathrm{ml}$ of venous blood radius (antecubital) was performed by an expert. Blood samples of 5 minutes at room temperature were immediately centrifuged. When the serum was separated, it was stored at $-20{ }^{\circ} \mathrm{C}$ for subsequent analysis(pars azmoon kit, iran). All measurements were performed by one person. The subjects sat on a chair and performed 50 eccentric contractions of 85\% 1RM in two groups. Each set includes 10 contractions that lowers a person's weight in 3 seconds and at least 2 seconds to reach the next contraction without weights placed in full flexion (Kasonuri Nosaka., 1995). One or three minute rest was given between each set. The experimental protocol was repeated at three consecutive days. All measurements except contusion, immediately before and after each training session.

\section{Result}

The result of ANOVA test showed that the mean of creatine kinase in the four groups was not significantly different at different stages of measurement $(p=0.67)$. Also, there is no significant difference between the mean of creatine kinase in the four groups $(\mathrm{p}=0.735)$ and the interaction between time and group is not significant ( $\mathrm{p}=0.329$ ) (Table 1 ).

Table 1: Analysis of repeated measurment variance of creatine kinase in four groups.

\begin{tabular}{|c|c|c|c|c|c|}
\hline Source & $\begin{array}{c}\text { Type III Sum } \\
\text { of Squares }\end{array}$ & Df & $\begin{array}{c}\text { Mean } \\
\text { Square }\end{array}$ & F & P \\
\hline time & 4084.680 & 3.193 & 1279.449 & 0.570. & 0.647 \\
\hline group & 51736.120 & 3 & 17245.373 & 0.427 & 0.735 \\
\hline interaction & 24852.480 & 9.578 & 2594.856 & 1.156 & 0.329 \\
\hline
\end{tabular}

The result of the analysis of variance showed that the mean of lactate dehydrogenase in the four groups of study was not significantly different in at least two stages of different stages of measurement $(\mathrm{p}=0.60)$. Also, there is no significant difference between mean of lactate dehydrogenase in the four groups 
$(p=0.901)$ and the interaction between time and the group is not significant $(\mathrm{p}=0.164)$ (Table 2).

Table 2: Analysis of repeated measurement variance of lactate dehydrogenase in four groups.

\begin{tabular}{|c|c|c|c|c|c|}
\hline Source & $\begin{array}{c}\text { Type III Sum } \\
\text { of Squares }\end{array}$ & df & Mean Square & F & P \\
\hline time & 35076.250 & 4 & 8769.062 & 2.319 & 0.060 \\
\hline group & 7005.180 & 3 & 2335.060 & 0.193 & 0.901 \\
\hline interaction & 64327.670 & 12 & 5360.639 & 1.418 & 0.164 \\
\hline
\end{tabular}

\section{Discussion}

There was no significant difference in the amount of creatine kinase in all stages of time and between different groups by manipulating the resting intervals and the number of setsrepetitions. The results of this study are in agreement with the results of Hotsun [2] and Chan [7] and are not consistent with the results of the trover [8] and Maywei [3]. According to Schwan's theory, the rupture and instability of muscle cell membranes result in the release of intracellular contents into intercostal water and ultimately of plasma, which is one of the possible mechanisms for increasing creatine kinase after eccentric exercises. The amount of Creatine kinase increases 24 hours after the eccentric exercise, which gradually reaches its maximum in 48 hours and returns to the baseline in 72 to 96 hours [4]. There is no significant difference in the plasma levels of creatine kinase. One of the interesting findings of the present study is that there is no significant difference between the different groups with rest periods and the number of different sets. Similarly, the reduction in creatine kinase is in the $1 \times 10 \times 5$ group. After the first stage, the trend was slowing down. Maeiyo et al. [3] reported that creatine kinase enzyme activity was more than a three minute interval after a resistance exercise with an intensity of $65 \%$, a maximum repetition of 10 sets with 10 repetitions, and a one minute resting distance. On the other hand, Rodrigo et al. [6] did not show any significant difference in activity of creatine kinase and lactate dehydrogenase enzyme after three sets and 10 repetitions. On the other hand, the response rate of creatine kinase depends on (repetition $\times$ sets / times) and the type of periodicity of rest the number of different responses [1]. One of the obvious reasons for the results of this study is the study by Maiyev et al. [3], mechanical load, and different muscle mass and type of exercise areas. In the present study, the protocol has been repeated for three consecutive days, but Miavi et al. [3] At a meeting held. There is also another possible reason for the subjects' training status, with the current protocol being implemented on untrained people, but previous studies have resisted practitioners [6], so that some kind of compatibility with practice repetition is observed in trained practitioners On the other hand, the reduction of plasma creatine kinase in the $1 \times 10$ $\times 5$ group, which after the first time showed a decreasing trend, is another interesting finding of the present study. The possible mechanisms for reducing creatine kinase can be attributed to the responsiveness of creatine kinase, which is influenced by individual factors and exercise variables such as genotype and high responsiveness, body composition and gender, mechanical and metabolic stress, workload amount of resting intervals Between turns shorter, the speed of movement, the involved muscle group, the age and type of muscle intramuscular, repetition eccentric exercise) $[5,4,2]$. In general, the significant difference between the different groups in the response to creatine kinase and the reduction of the response of creatine kinase after the first phase of externally exercises implies that apart from the resting intervals between the turns and the number of repetitive sets, important variables There is another that affects the response to muscle damage indices. On the other hand, there was no significant difference in the level of lactate dehydrogenase at any stage of time and also among all groups. Lactate dehydrogenase increased after the first time and showed a decreasing trend after the last training. In the 24 hours after the last exercise, in the 4 groups showed an increasing trend. The results of this research are consistent with the results of the trover research [8] and are not consistent with the results of the research Arazi [1] and Mayaevo [3].

Possible mechanism of increasing lactate dehydrogenase after mechanical stress (decomposing sarcomere and components of $\mathrm{z}$ ) and metabolic pathways (increasing the permeability of the membrane of the cell and thereby increasing the calcium ion, which is the activity of potassium channels and protein degrading enzymes).However, the reduction of lactate dehydrogenase after the first time, after the last one, which is one of the interesting findings of the present study and contradictory with $[1,3]$, is due to the fact that the response of lactate dehydrogenase, such as creatine kinase is influenced by, Duration of exercise, nutrition and supplements, the number of movements and muscle groups involved $[4,6,7,9]$.

\section{Conclusion}

In general, the results of this study showed that repetition of eccentric exercise during three consecutive days increased muscle damage and did not result in adaptation, which is independent of the difference in rest intervals between sets.

\section{References}

1. Arazi H, Rahimi R (2011) The effect of different rest intervals between multiple bench press bouts. South African Journal for Research in Sport, Physical Education and Recreation 33(1): 1-8.

2. Howatson K, Someren V, Hortobágyi T (2007) Repeated Bout Effect after Maximal Eccentric Exercise. Int J Sports Med 28(7): 557-563.

3. Mayhew D, Thyfault J, Koch A (2005) Rest-interval length affects leukocyte levels during heavy resistance exercise. J Strength Cond Res 19(1): 16-22.

4. Mohammadi H, Sahebazamani M, Ghahraman TK (2015) The effect of repeated bouts of eccentric exercise on some of Biochemical markers of Delayed onset muscl soreness. International Journal of Applied Exercise Physiology 4(1): 1-2.

5. Pereira R, Machado M (2007) Resistance exercise- induced microinjuries do not depend on 1 or 3 minutes rest time interval between series. International Journal of Science Issn 4(4): 1885-3137. 
6. Rodrigues BM, Dantas E, Salles BF, Miranda H, Koch AJ, et al. (2010) Creatine kinase and lactate dehydrogenase responses after upper body resistance exercise with different rest intervals. J Strength Cond Res 24(6): 1657-1662.

7. Chan R, Newton M, Nosaka K (2011) Effects of set-repetition configuration in eccentric exercise on muscle damage and the repeated bout effect. Eur J Appl Physiol 112(7): 2653-2661.
8. Trevor C, Hsieh SS (2001) Effects of a 7-days eccentric training period on muscle damage and inflammation. Med Sci Sports Exerc 33(10): 1732-1738.

9. Willardson JM (2006) A brief review: factors affecting the length of the rest interval between resistance exercise sets. J Strength Cond Res 20(4): 978-984.

\section{Your next submission with Juniper Publishers will reach you the below assets}

- Quality Editorial service

- Swift Peer Review

- Reprints availability

- E-prints Service

- Manuscript Podcast for convenient understanding

- Global attainment for your research

- Manuscript accessibility in different formats

( Pdf, E-pub, Full Tsext, Audio)

- Unceasing customer service

Track the below URL for one-step submission https://juniperpublishers.com/online-submission.php 